\title{
Chapter
}

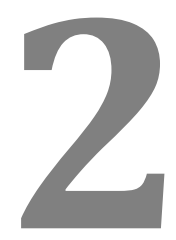

\section{DIAGNOSIS OF COMMUNICABLE DISEASES USING PAPER MICROFLUIDIC PLATFORMS}

Sanjay Kumar, Pulak Bhushan, and Shantanu Bhattacharya*

Microsystems Fabrication Laboratory, Department of Mechanical Engineering, Indian Institute of Technology, Kanpur, India 


\section{Contents}

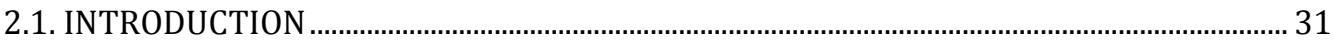

2.2. BASICS OF PAPER-BASED MICROFLUIDIC PLATFORMS ….................................................. 32

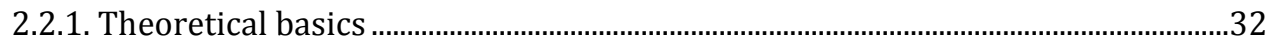

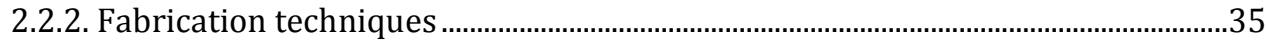

2.2.3. Working principle of a paper-based microfluidic device..........................................36

2.3. PAPER-BASED MICROFLUIDIC PLATFORMS FOR DIAGNOSIS OF

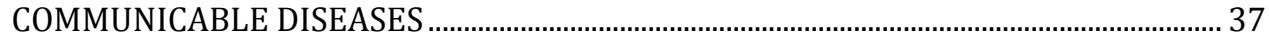

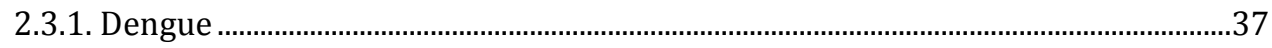

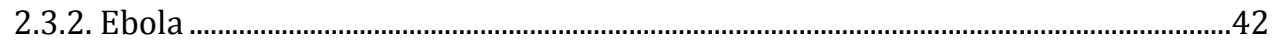

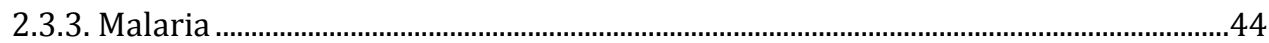

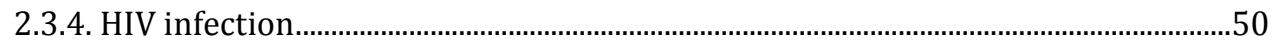

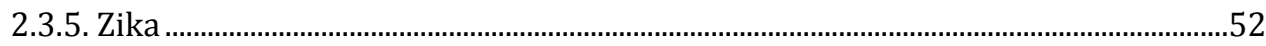

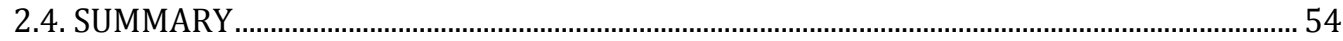

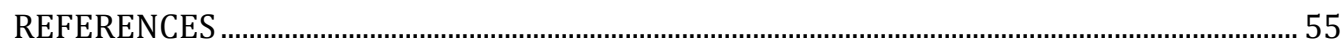




\subsection{INTRODUCTION}

A communicable disease is an infection spread from person to person or from people to animals and vice versa. The infection is transmitted through air, through direct contact with blood, faeces or other bodily fluids of an infected individual or indirectly through a vector [1]. Based on the agent causing the disease (including bacteria, viruses and parasites), there are nine broad classifications of communicable diseases: viral diseases (dengue, Ebola, human immunodeficiency virus (HIV) and Zika), rickettsial diseases (rickettsial pox, rocky mountain spotted fever, trench fever), mycoplasmal diseases (mycoplasma pneumonia), chlamydial diseases (trachoma), bacterial diseases (typhoid, pneumonia, cholera, tuberculosis), spirochaetal diseases (syphilis), protozoan diseases (malaria, amoebiasis, diarrhoea, kala-azar), helminthic diseases (ascariasis) and fungal diseases (ringworm or tinea) [2]. Among these, viral and protozoan diseases are infections that are extremely widespread. Viral diseases are caused by invasion of the human body by viruses (microorganisms) [3]. There are a variety of viruses that cause various viral diseases. Viral diseases show several types of symptoms with varied characteristics whose severity depends on the type of infection. They typically last for long periods of time inside the body, hence antibiotics alone are not sufficient for treating them $[1,4]$. Protozoa are one-celled microorganisms that may be free-living or parasitic in nature, causing diseases in humans. Protozoa multiply very rapidly once they enter the human body, causing serious threats to human life. Infections can be transmitted from one body to another through various routes such as the faecal-oral route, through direct contact between people or by an arthropod vector (e.g. the bite of a mosquito or sand fly). Protozoan diseases such as malaria, trypanosomiasis, toxoplasmosis and cryptosporidiosis pose severe danger to humans and have been responsible for more than a million deaths annually worldwide [5].

Communicable diseases mostly spread in developing countries like African countries, India, Bangladesh, etc. Due to a lack of proper diagnostic and treatment facilities, these diseases are the cause for over $95 \%$ of the deaths in these countries [6]. Insufficient skilled personnel, poor emergency medical care facilities, limited infrastructure, few resources and poor economic conditions are some of the reasons responsible for the poor healthcare services in these countries. Owing to this, timely and rapid diagnosis of diseases like malaria, dengue, Ebola, etc. is imperative. A major challenge and opportunity for the research community is to develop rapid diagnostic tools that can help in prevention of such diseases. The World Health Organization (WHO) has established a set of guidelines for the development of rapid diagnostic tools, i.e. ASSURED (affordable, sensitive, specific, user-friendly, rapid and robust, equipment-free and deliverable to users). 
Paper-based lateral flow assays are suitable for point-of-care (POC) rapid diagnosis of diseases. Paper-based rapid diagnostic tests (RDTs) have several advantages: they are thin, lightweight, disposable, can be easily modified chemically, are compatible with many bioassays and have no requirement for extra instrumentation in diagnosis.

This chapter initially elaborates on the theoretical basics and various fabrication techniques of paper-based microfluidic platforms. Dengue, Ebola, malaria, HIV infection and Zika are some of the disease entities that have been reported to have high fatality rates. Delayed diagnosis of such diseases presents the disease in a more acute and problematic way for case management. In the later section, a detailed review of the various paper-based microfluidic platforms developed for the diagnosis of the above diseases is provided.

\subsection{BASICS OF PAPER-BASED MICROFLUIDIC PLATFORMS}

In this section an attempt has been made to explain the theoretical basics of paper-based microfluidic platforms by investigating capillary-driven flows that are generally observed in paper matrices with varying pore size distributions. Also, various fabrication techniques which are involved in the realization of such paper-based platforms are discussed at length. The section concludes with the basic working principles of paper-based platforms including the different detection methodologies.

\subsubsection{Theoretical basics}

Lateral flow assays are performed using paper strips made of nitrocellulose membrane. The fluid flows through the strip under capillary action. When a paper strip is dipped in a liquid reservoir, a pressure gradient is created at the interface of the dry and the wet side of the paper. This pressure difference is the fundamental driving force for the capillary flow $[7,8]$.

Fluid flow through a uniform cross section of the paper strip. In a uniform cross-sectional area of the paper strip (Figure 1), the capillary suction pressure is given by [9]:

$$
P_{\mathrm{c}}=\frac{2 \gamma \cos \theta}{r_{\mathrm{a}}}
$$

where $\gamma$ is the interfacial surface tension, $\theta$ is the contact angle of the liquid with the paper strip and $r_{\mathrm{a}}$ is the average pore radius.

The pressure difference over the wetted region of the paper strip of a certain length $l$ is given as:

$$
\Delta P=P(l)-P(0)
$$




$$
\begin{aligned}
& \Delta P=P_{\mathrm{atm}}-P_{\mathrm{c}}-P_{\mathrm{atm}} \\
& \Rightarrow \Delta P=-P_{\mathrm{c}}=\frac{-2 \gamma \cos \theta}{r_{\mathrm{a}}}
\end{aligned}
$$

The negative sign in equation (2) implies that the pressure decreases with the distance as the fluid moves in a given direction.
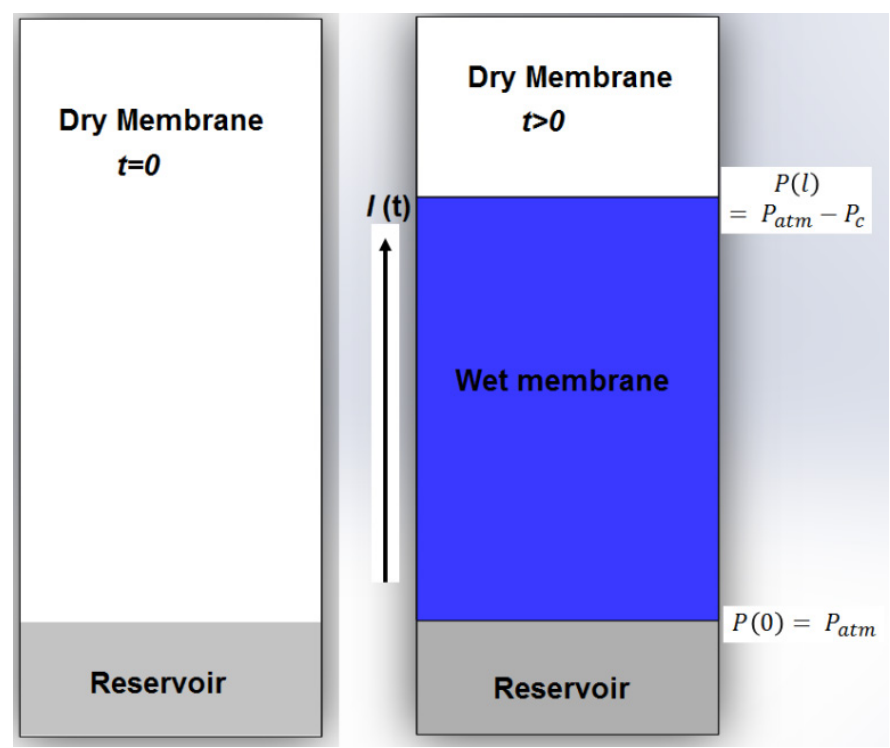

Figure 1. Schematic of fluid flow through paper strip (uniform cross section)

The rate of movement of the liquid front in a homogeneous porous medium of uniform dimensions is governed by Darcy's law as follows:

$$
\hat{u}=\frac{k_{\mathrm{i}} \Delta P}{\mu L_{\mathrm{w}}}
$$

where $k_{\mathrm{i}}=\frac{k}{\varnothing}$ is the interstitial permeability of the paper strip, $\emptyset$ is the porosity of the medium, $\mu$ is the fluid viscosity, $L_{\mathrm{w}}$ is the length of the wetted region of the paper and $\Delta P$ is the pressure difference over the wetted region.

In the case of capillary-driven flow, the pressure difference is dependent on time, i.e. the fluid flow is unsteady uniform [10]. The position of the fluid front with respect to time can be modelled by the Lucas-Washburn equation [11].

For 1-D fluid flow, equation (3) can be written as

$$
\Rightarrow l . d l=\frac{k \Delta P}{\emptyset \mu} d t \quad \frac{d l}{d t}=\frac{k_{\mathrm{i}} \Delta P}{\mu l}
$$


Integrating equation (4) we have:

$$
\begin{aligned}
& \int_{l=0}^{l=l(t)} l . d l=\frac{k \Delta P}{\emptyset \mu} \int_{t=0}^{t} d t \\
& \Rightarrow l(t)=\sqrt{\frac{k \Delta P}{\emptyset \mu}} \sqrt{t}
\end{aligned}
$$

On substituting the value of $\Delta P$ from equation (2) we get:

$$
\Rightarrow l(t)=2 \sqrt{\frac{k \gamma \cos \theta}{\emptyset r_{\mathrm{a}}}} \sqrt{t}
$$

Fluid flow through a varying cross section of the paper strip. Darcy's law and the Lucas-Washburn equation are not valid in the case of fluid flow through an area of varying cross section. Considering fluid flow in a paper strip with a sudden change in its cross section, as shown in Figure 2(a), where the fluid flows from section 1 (uniform cross section) to section 2 (varying cross section), when the liquid front reaches the plane AA, it encounters a sudden increase in the number of pores to be filled by it (i.e. a continuous increase in the available bed volume).

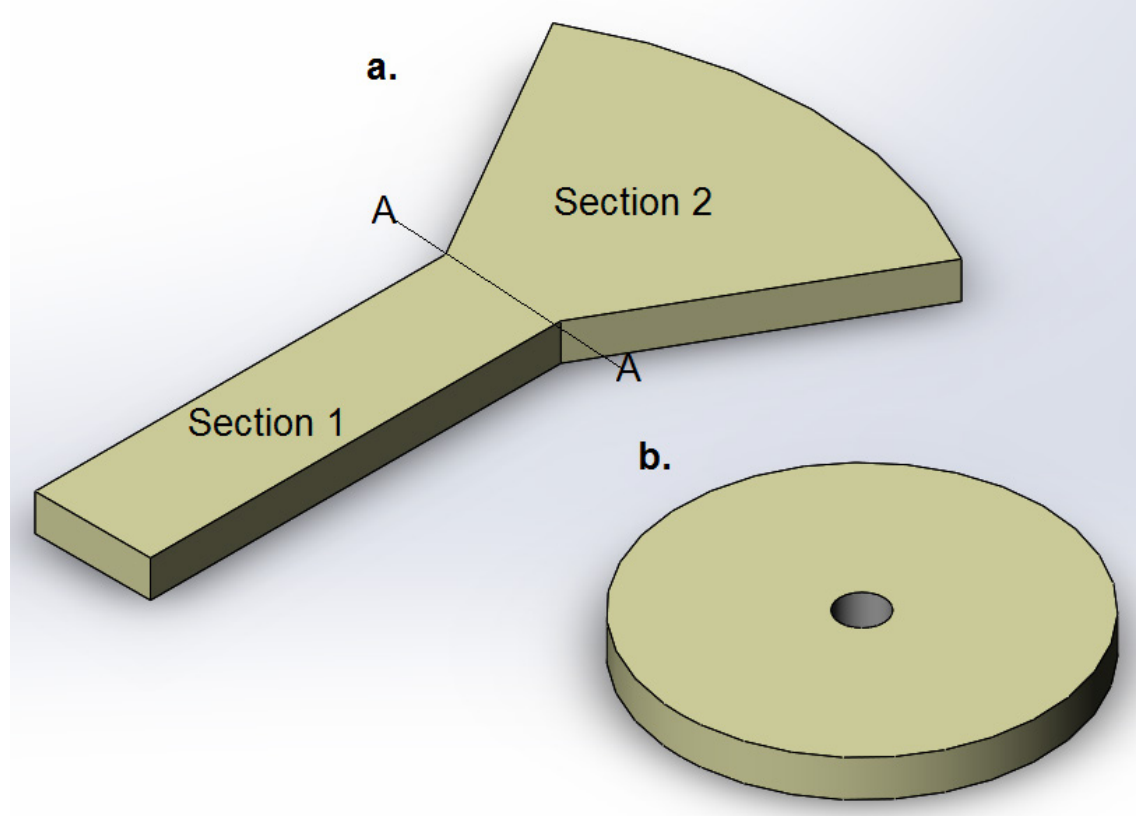

Figure 2. Schematic of paper strips with varying cross section.

(a) rectangular with fan, (b) circular section 
The volumetric fluid flow rate for the fan portion of the paper strip shown in Figure 2(a) is given as [12]:

$$
Q=\frac{k \Delta P}{\mu \int_{l}^{l_{f}=(L-l)} A(x)}
$$

where $A(x)$ is the area of the varying cross section, $l$ is the length of section 1 , $l_{\mathrm{f}}$ is the length of section 2 and $L$ is the total length of the paper strip.

Also, the volumetric flow rate for a circular paper strip (Figure 2(b)) can be predicted by the following relationship $[13,14]$ :

$$
Q=\frac{2 \pi K h \gamma h_{\mathrm{eq}} \cos \theta}{3 \mu \ln \left(\frac{2 r}{b}\right)^{2}}
$$

where $h$ is the thickness of the paper, $r$ is the wicking radius, $b$ is the initial diameter of the fluid droplet, $h_{\mathrm{eq}}$ is the equivalent thickness of the radial capillary, $\theta$ is the wetting angle between the liquid sample and the cellulose fibres and $K$ is the parameter for moisture content in the paper.

\subsubsection{Fabrication techniques}

A number of fabrication techniques have been explored by researchers to fabricate paper-based detection devices. They can be divided into two broad categories: devices made by cutting into desired shapes and sizes and devices made by creating hydrophobic barriers on the paper strip to manipulate the direction of the fluid. Paper-based devices are generally fabricated by cutting, since it is chemical-free, inexpensive and a single-step process. Scissors and paper cutters are the general way of cutting paper, but they are prone to uneven cutting due to manual handling [15]. Instead, $\mathrm{CO}_{2}$ laser beam machining is used for precise and selective cutting of paper strips [16]. There are several advantages associated with the cutting process such as rapid fabrication, no requirement for additional material for creating hydrophobic barriers and control of fluid flow by cutting the paper strips into optimized shapes. As a replacement for cutting, patterns are made on a paper device by depositing hydrophobic materials to define the shape (i.e. width and length) of the device. The conventional methods used for creating hydrophobic barriers are inkjet printing [17-19], wax printing [20,21], flexographic printing [22], photolithography [23], wax dipping [24], plasma treatment [19,25], screenprinting of poly(dimethylsiloxane) (PDMS) [15], lacquer spraying [26] and using a permanent marker [27]. The details of these fabrication methods have been studied comprehensively by several researchers $[11,28,29]$. The selection of fabrication methodology is primarily dependent on the nature of the reagents used and the resolution required by the device [11]. 


\subsubsection{Working principle of a paper-based microfluidic device}

In recent years, numerous techniques have been used for detection of biochemical assays on paper-based devices such as colorimetric, electrochemical, fluorescence, chemiluminescence, electrochemiluminescence, etc. [28,30]. Colorimetric detection is one of the most widely accepted detection techniques for diagnostics. It is based on observation of a change in colouration by the naked eye so that no extra instrumentation is required to perform the test.

Colorimetric paper-based microfluidic devices. Colorimetric detection is an extensively used technique for the diagnosis of communicable diseases using paper-based platforms. In a typical colorimetric detection assay, five major zones are present: sample pad, conjugate pad, test line, control line and absorbent pad (Figure 3). Human blood, plasma or serum is loaded onto the sample pad after which it migrates to the conjugate pad (CP). On the CP, conjugates of nanoparticle (NP) and antibody (Ab) (e.g. gold/silver NP-Ab) are pipetted and dried. It has been reported that the colour of the nanoparticles is dependent upon their size/shape, e.g. the colour of silver nanoparticles (AgNPs) changes from yellow to green with an increase in their diameter [31]. Therefore, on conjugation of NPs with Abs, the mean hydrodynamic size of the NPs increases and exhibits a change in colour. When the sample liquid reaches the $\mathrm{CP}$, the antigens present in the sample bind with the conjugated NP-Ab to form NP-Ab/antigen complexes. These complexes move ahead towards the test line by capillary action through a nitrocellulose membrane. The test line contains immobilized antigen on it. If the antigen is present in the sample then there are no unoccupied antibodies left (ones that are conjugated to the nanoparticles) and there will be no colour change at the test line, whereas if antigens aren't present in the sample, the antigens embedded on the test line immobilize on the antibodies resulting in a colour change at the test line. The control line is loaded with secondary antibodies specific to the antibodies conjugated to the NPs. Here, the conjugated NP-Ab binds with secondary antibodies to demonstrate a colour change (in both cases when antigen is present in the sample or not). The change in colour at the control line confirms the successful completion of the colorimetric assay. The role of the absorbent pad is to soak up the extra liquid. Similarly, detection can also be performed by embedding antibodies on the test line instead of antigens. After the NP$\mathrm{Ab} /$ antigen complexes reach the test line, if an antigen is present in the sample then the antibodies on the test line conjugate with the complex making a sandwich structure (antibody-antigen-antibody). A colour change can then be observed at the test line. Whereas, if antigens aren't present in the sample, no sandwich structure is formed resulting in no colour change at the test line. Accordingly, depending on the entity embedded on the test line (antigen/antibody) a change in colour can signify a negative or a positive result. 


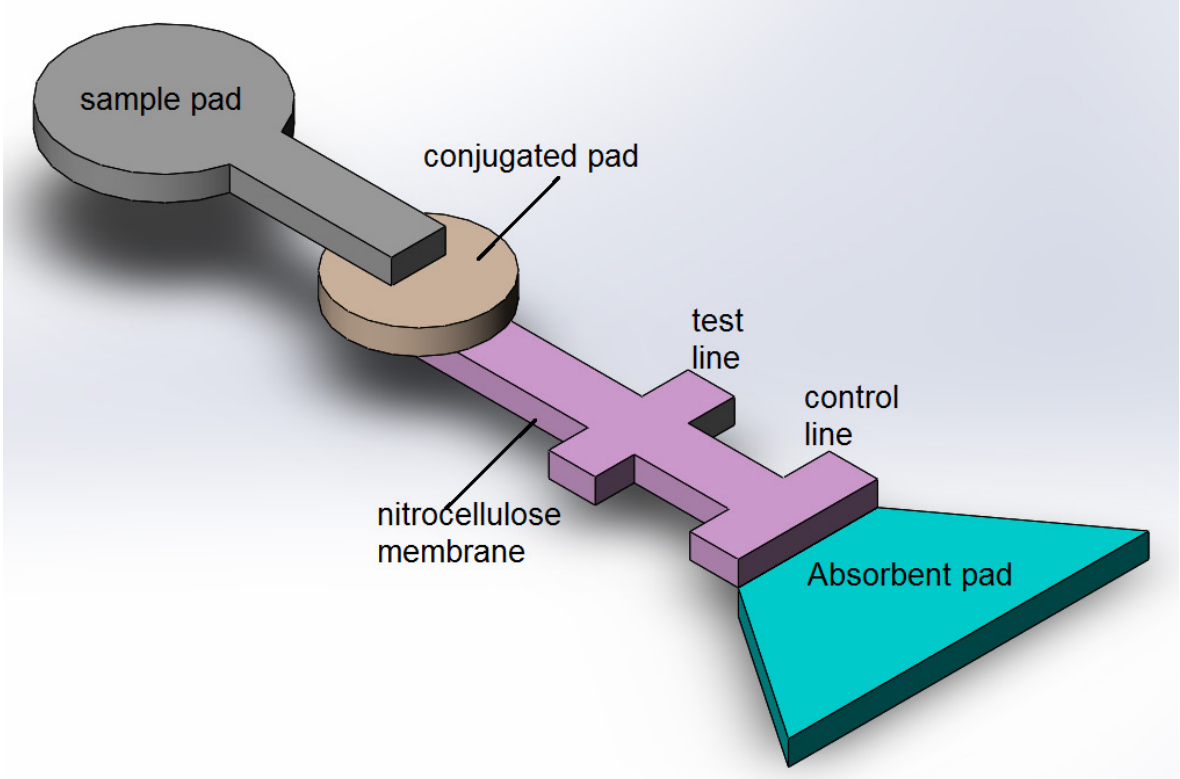

Figure 3. Schematic of a paper device based on a colorimetric assay

\subsection{PAPER-BASED MICROFLUIDIC PLATFORMS FOR DIAGNOSIS OF COMMUNICABLE DISEASES}

Paper is a ubiquitous material that has proved to be promising for developing microfluidic platforms for the detection of communicable diseases. It offers simple and cheap technology that is easy to use, requires minute amounts of sample and can be easily disposed of through incineration. Extensive research is being carried out in this field owing to these unique properties of paper. This section reviews the various paper-based platforms fabricated to date for detection of several communicable diseases. In addition to that, the advantages and gaps that still need to be tackled are highlighted as well.

\subsubsection{Dengue}

Dengue is one of the most life-threatening vector-borne viral diseases, spreading rapidly all over the world. It occurs mainly in tropical and subtropical regions, mostly in urban and semi-urban areas [32]. It is spread primarily in the Pacific, Southeast Asia and the Americas. In India, the number of patients infected with dengue fever has been growing rapidly (Figure 4). 
India contributes around $19 \%$ of the total dengue cases in South-East Asia Region (SEAR) countries [33].

Dengue is a flavivirus that belongs to the family Flaviviridae. Dengue virus (DENV) is a small RNA (single-stranded, spherical in shape with a diameter around $50 \mathrm{~nm}$ ) virus. The virus contains a membrane bilayer (derived from the host) and a single copy of the RNA genome. Three structural proteins are encoded by $11 \mathrm{~kb}$ nucleotides: the capsid (C), membrane (M) and envelope (E) glycoproteins and there are seven non-structural proteins (NS1, NS2A, NS2B, NS3, NS4A, NS4B and NS5) [32,34,35]. There are four distinct but antigenically related serotypes of the virus that causes dengue, viz. DENV1, DENV2, DENV3 and DENV4 [36]. Infection by any of these serotypes may cause illnesses in individuals, encompassing classical dengue fever (DF), fatal dengue haemorrhagic fever (DHF) and dengue shock syndrome (DSS) [36-39]. Fever, headache, weakness, rash and body aches are common symptoms of classical DF. DHF is a severe form of DF that tends to affect children under the age of ten. Severe pain in the abdomen, bleeding from the nose and the mouth, frequent vomiting and restlessness are symptoms of DHF. DSS is characterized by plasma leakage that may appear as tiny blood spots or large blood patches on the skin.

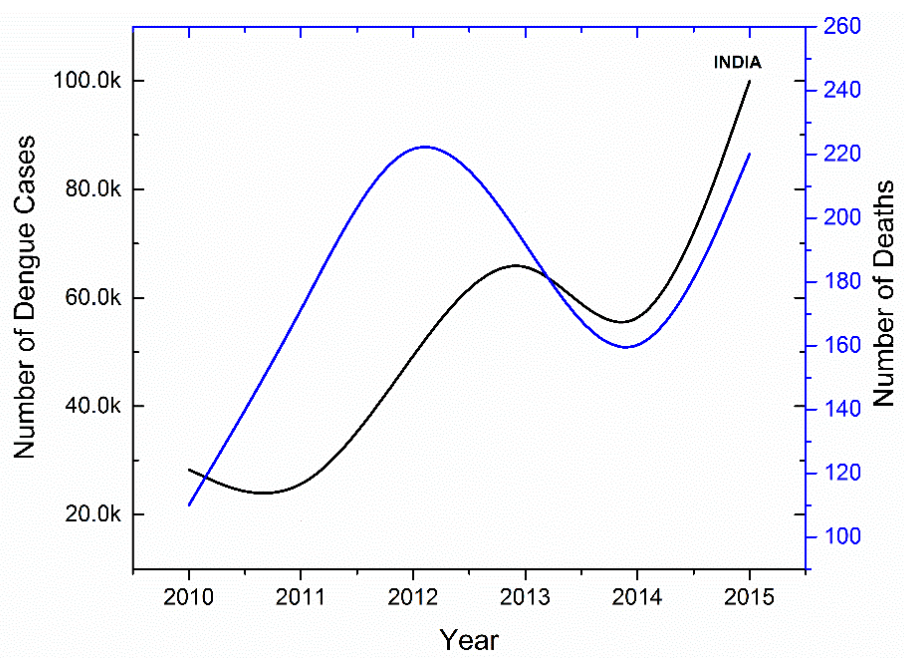

Figure 4. Dengue cases in India. Source: National Vector Borne Disease Control Programme, New Delhi, India.

Causative agent. DF is a viral disease caused in humans by a bite from an infected female mosquito (either Aedes aegypti or A. albopictus). A. aegypti, a principal vector of dengue, is an aggressive biter (bites generally in the early morning or evening). It lives in dark indoor areas, e.g. closets, bathrooms and under beds. A. albopictus, the second mosquito species also known as the Asian 
tiger mosquito, lives in rainwater storage areas. This mosquito can survive in cold temperatures unlike A. aegypti. When these mosquitoes bite humans, the virus incubates for 3-14 days before the first symptoms appear.

Paper-based microfluidic platforms for its diagnosis. Generally, DENV is diagnosed using several approaches such as virus detection (cell culture, immunofluorescence), viral RNA detection, antigen detection (NS1-based assays, immunohistochemistry), dengue antibody detection (hemagglutination inhibition, enzyme-linked immunosorbent assay (ELISA), complement fixation test), nucleic acid detection (real-time reverse transcription polymerase chain reaction (RT-PCR)) and serological methods (IgM- and IgG-based assays) $[37,39]$. These laboratory-based diagnostic methods are highly specific and essential for precise diagnosis of dengue infection, but they are expensive and need highly clean labs and expert human skills to perform. Therefore, paperbased RDTs can be useful as dengue diagnostic tools in rural areas or areas with inadequate resources. Paper-based devices are largely based on either antigen detection or antibody detection. In a typical paper-based dengue detection system, when an infected blood sample containing either nonstructural protein 1 (NS1) or dengue IgM or IgG is added to the sample pad, it combines with conjugated gold NP-Ab (specific) on the $\mathrm{CP}$ and forms antibody-antigen complexes. These complexes are captured by the respective secondary antibodies embedded on the test line resulting in a colour change at the test line.

In the past several years, paper-based lateral flow assays have been used for screening of dengue patients. A stacking flow platform has been developed by Zhang et al. [40] for detection of dengue-specific IgG in salivary fluid. The stacking flow device comprises a test strip (made of nitrocellulose membrane), a sample pad (made of glass fibre), an absorbent pad (made of cellulose) and a flow regulator. A schematic diagram of the stacking lateral flow immunoassay is shown in Figure 5. The flow regulator (made by stacking two paper strips over each other) is used for the proper mixing of reagents and ensuring uniform fluid flow through the strip. The glass fibre sample pad helps reduce interference by removing non-desirable salivary substances. As a result, the gold nanoparticles adhere to the specific antibody-antigen only, improving the device sensitivity. This device is able to detect dengue IgG within 20 min with a limit of detection of $20 \mathrm{ng} \mathrm{mL}-1$. 


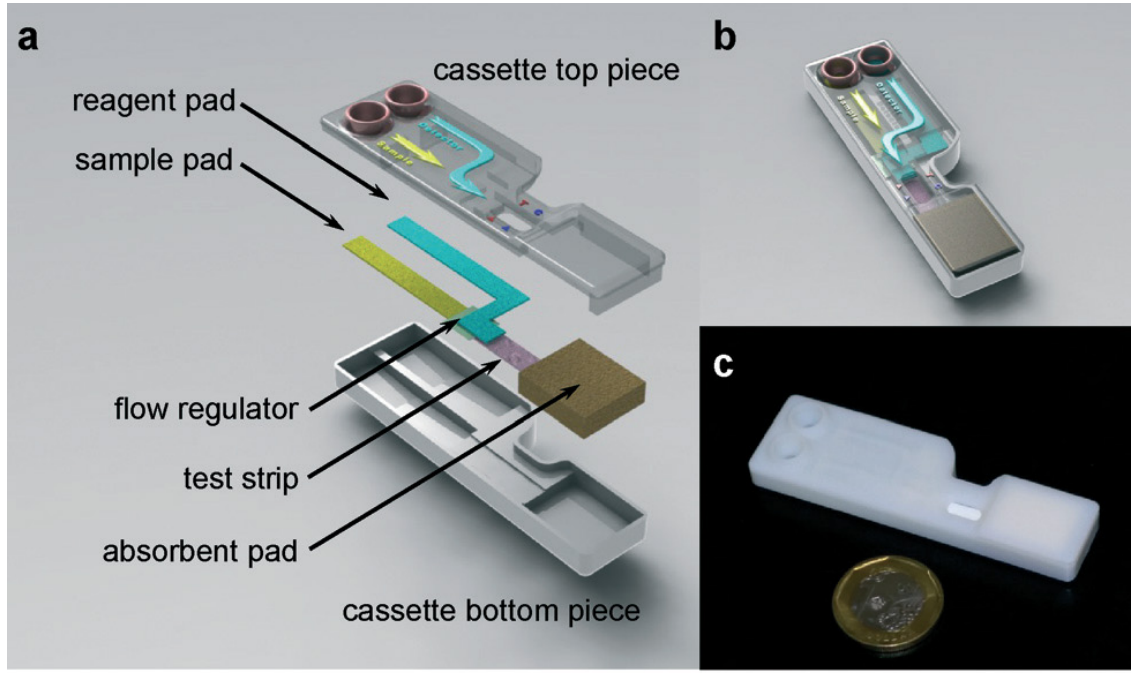

Figure 5. Schematic of a stacking flow immunoassay. Reprinted with permission from Zhang et al. [40]. ${ }^{\odot 2016}$ Royal Society of Chemistry.

Lo et al. [35] developed an in vitro diagnostic device for nucleic acid detection of dengue virus serotype-2 (DENV2) RNA. The device combines a paper-based diagnostic platform with reverse transcription loop-mediated isothermal amplification (RT-LAMP). Nucleic acid amplification is done using LAMP to achieve high specificity and sensitivity which helps in accurate detection of DENV. A paper-based strip containing 96-well plates is printed via simple wax printing, thereby forming individual test zones. The detection of DENV2 RNA is carried out in two steps: firstly, DENV2 RNA is reverse transcribed and amplified via RT-LAMP for $1 \mathrm{~h}$ (single-stranded RNA converted into doublestranded DNA). In the second step, fluorescent measurement of the RT-LAMP products is carried out using DENV2-specific fluorescent probes in paperbased test zones. A schematic diagram of the detection methodology is shown in Figure 6. The detection time of this device is reported as $100 \mathrm{~min}$. 
(a)

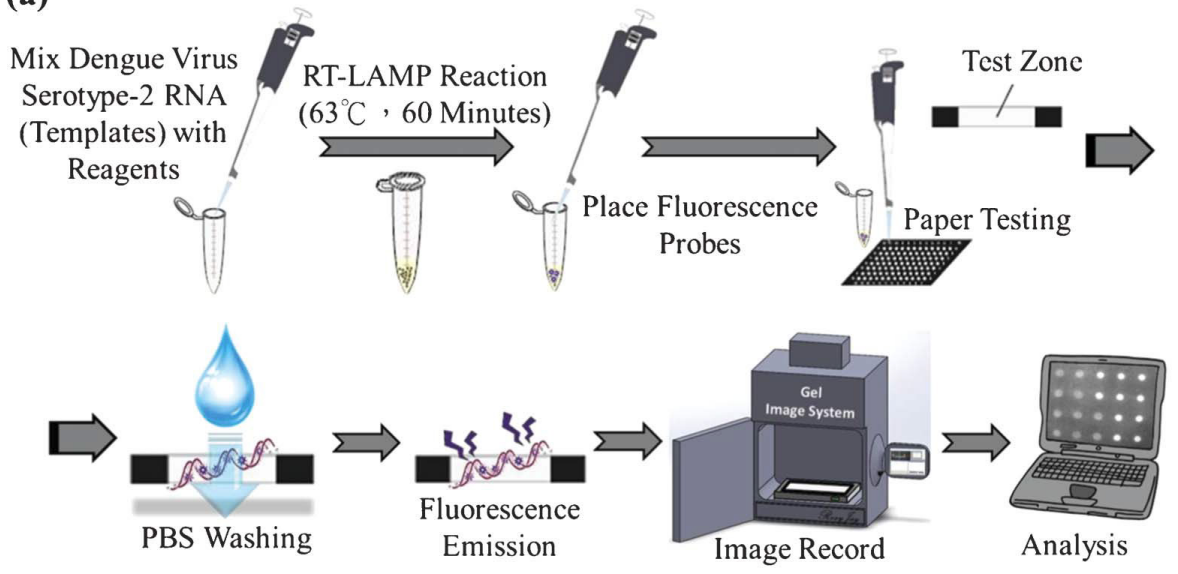

(b)
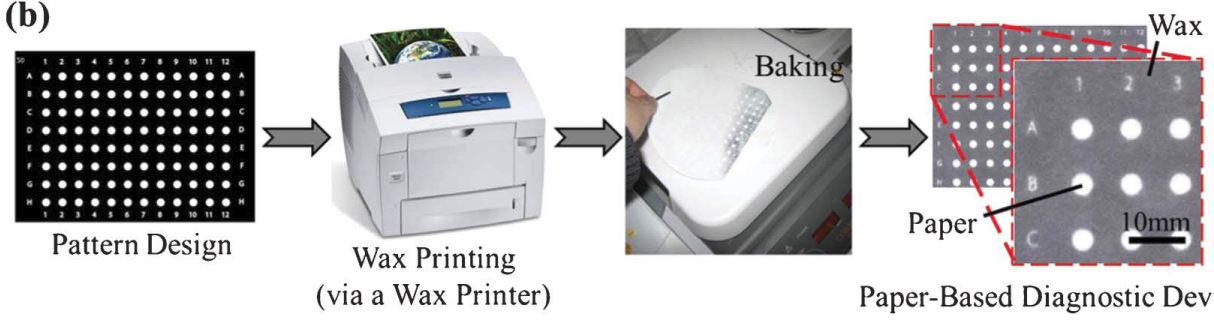

Paper-Based Diagnostic Device

Figure 6. Schematic of a paper-based device fabrication and detection procedure for detection of DENV2. Reprinted with permission from Lo et al. [35]. ${ }^{\circ 2016}$ Royal Society of Chemistry.

Yager et al. [41] reported a two-dimensional paper network for detection of IgM and IgG antigens. The paper device was made by the origami method (i.e. folding of paper segments). Reagent flow through the paper strip is controlled by placing dissolvable sugar barriers. It was reported that addition of sucrose to the nitrocellulose membrane results in a reduction of flow rate. In order to improve the screening of dengue disease in remote locations, a dengue detection image processing algorithm has been developed and embedded in smartphones [42]. This technique was developed to help patients transfer the results from the device to the Centre for Disease Control (CDC) for further analysis to aid patients' online monitoring. A schematic diagram of the hybrid dengue diagnostic system is shown in Figure 7. 


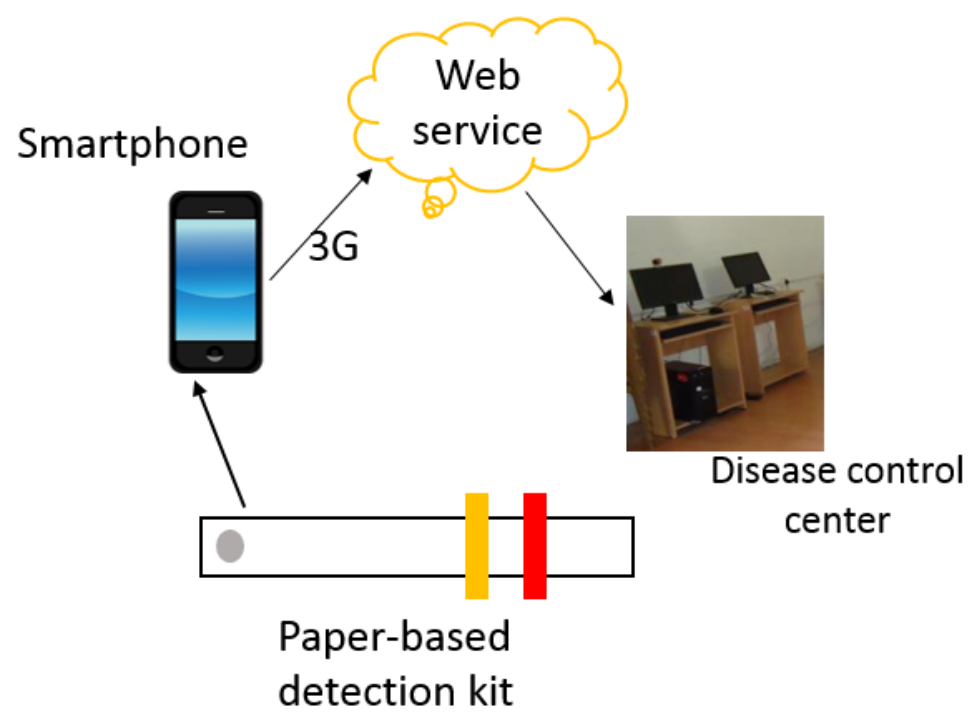

Figure 7. Schematic of a mobile dengue detection system

There are several commercial dengue detection kit manufacturers available in the Indian market such as SD Bioline, Accuquik, Shree Shyam Bio-medicals, Oscar Medicare Pvt. Ltd., Mediclone Biotech Pvt. Ltd., etc. The main concerns of these available devices are low sensitivity and specificity. Wang et al. [39] performed numerous tests on the SD Bioline commercial kit using human blood samples. The sensitivity of these kits was found to be approximately $88.65 \%$. Despite the efforts made in the development of rapid detection kits for dengue, some issues still need to be resolved, e.g. device sensitivity, simultaneous detection of multiple viral fevers, device robustness and fabrication cost.

\subsubsection{Ebola}

Ebola is a viral haemorrhagic fever which is a highly infectious and deadly disease, first discovered in 1976 in Zaire. It is an animal-borne virus hosted predominantly in fruit bats [43]. Direct contact with blood or body fluids (i.e. saliva, urine, semen, etc.) of an infected person is the means by which the virus spreads in humans [44]. The virus incubates for a period of around 2-21 days. Initial indications of the symptoms include fever, fatigue, myalgia and neurological manifestations. It is then followed by nausea, vomiting, diarrhoea, impairment of liver and kidney function and internal bleeding [45].

Causative agent. It is caused by the genus Ebolavirus belonging to the Filoviridae family. There are five primary species of Ebolavirus, namely Taï Forest ebolavirus, Bundibugyo ebolavirus, Zaire ebolavirus, Sudan ebolavirus and Reston ebolavirus. The first four species are known to cause infections in 
humans, whereas the fifth virus has caused infections among nonhuman primates [45].

Paper-based microfluidic platforms for its diagnosis. Ebola is generally diagnosed using RT-PCR [46], antibody-capture ELISA [47], antigen-capture detection tests and virus isolation through cell culture. Despite the fact that these methods are highly sensitive and specific, they suffer from issues like poor portability, being highly time consuming and requiring laboratory equipment. Major outbreaks of Ebola can be managed by using rapid, specific and highly sensitive portable sensing systems operable in low-resource areas, e.g. paper-based assays.

Pardee et al. have developed an in vitro paper-based diagnostic platform for Ebola virus (EBOV) [48]. The sensor detects the virus by utilizing synthetic gene circuits coupled with visual detection using colorimetric outputs. The gene networks embedded onto the paper platform respond to small molecules and synthetic RNA. Sudan and Zaire strains were used to test the specificity of the developed assay and it was found to have sensitivity at a concentration of $30 \mathrm{nM}$ trigger RNA for both strains. The technology demonstrates successful detection of EBOV by exploiting gene circuits, genetic switches and programmable in vitro diagnostics. Using multi-coloured AgNPs Yen et al. have developed a low-cost paper-based platform for multiplexed pathogen detection [31]. The assay is capable of detecting DENV, EBOV and yellow fever virus (YFV) simultaneously. AgNPs of varying sizes and triangular shape are conjugated with antibodies binding to specific biomarkers only, thus using the colour of the nanoparticle to distinguish between the three pathogens. Conjugation of orange, red and green AgNPs to anti-YFV NS1 monoclonal antibody, anti-EBOV GP monoclonal antibody and anti-DENV NS1 monoclonal antibody, respectively, is performed. Figure 8 shows a schematic of the designed lateral flow assay and the three pathogens being distinguished on it. First, the three conjugated antibodies are loaded onto the paper on three individual detection lines, and the green, red and orange signals are observed at the detection line where the respective antibodies are immobilized (Figure 8 (a)). Next, all three antibodies for the three diseases are mixed in equimolar concentrations and printed as a single detection line on the test strip. The detection line shows the presence of YFV, EBOV and DENV by turning orange, red or green according to the pathogen present in the human serum (Figure 8(b)). 


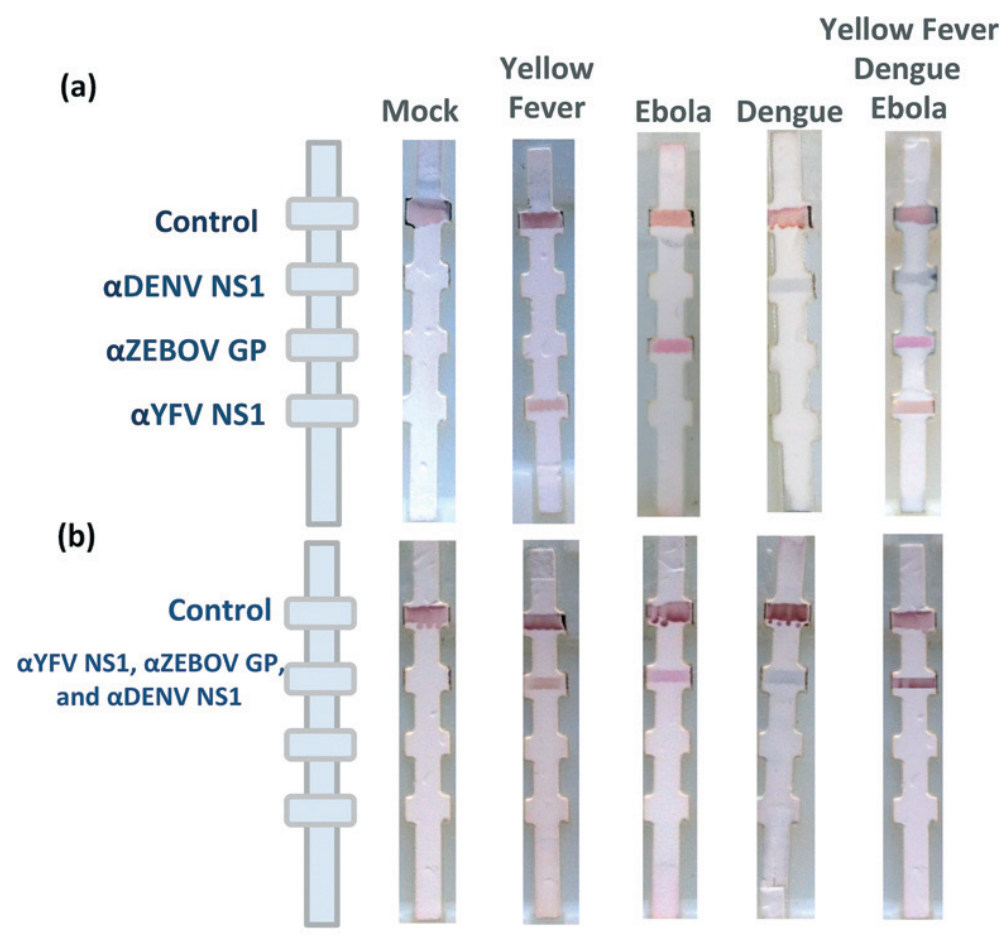

Figure 8. Multiplexed detection using AgNPs. (a) Colours form corresponding to the antigen present in the sample, (b) mixed antibodies printed onto a single test line. Different colours exhibited: YFV: orange; EBOV: red; DENV: green. Reprinted with permission from Yen et al. [31]. ๑2016 Royal Society of Chemistry.

The limit of detection of the assay is reported to be as low as $150 \mathrm{ng} \mathrm{mL}-1$. The device demonstrates no cross-contamination, high specificity and high sensitivity towards the three pathogens. An antigen rapid test kit has been manufactured by Corgenix Medical Corporation, USA for detection of EBOV VP40 antigen. It is an immunochromatographic dipstick immunoassay capable of detection using the whole blood, serum or plasma of an individual [49]. The sample is added to the sample pad then the dipstick is inserted into a tube containing sample buffer to initiate sample flow. The antigens present in the sample form immune complexes on coming into contact with the antibodies conjugated to gold nanoparticles. A change in colour to pink-red confirms the presence of EBOV in the sample. The fabricated device gives a result within $15 \mathrm{~min}$ and shows sensitivity and specificity of $91.8 \%$ and $84.6 \%$, respectively.

\subsubsection{Malaria}

Malaria is a life-threatening blood disease caused by the bite of an infected Anopheles mosquito vector. There are five types of Plasmodium parasite that 
infect humans, Plasmodium falciparum, $P$. ovale, $P$. vivax, $P$. knowlesi and $P$. malariae [50]. P. falciparum and P. vivax pose the greatest threat to humans worldwide.

Causative agent. Malaria is also a parasitic disease. After inoculation into a human by a female Anopheles mosquito, the parasite reaches the liver via the bloodstream, where it matures. Subsequently, the parasites infect the red blood cells (RBCs) in several ways, like destroying the RBCs and increasing the viscosity of the cells. Malaria is caused by five species of parasite belonging to the Plasmodium genus, of which P. falciparum is the most widespread and fatal [51]. P. falciparum is responsible for changing the shape of an RBC from a double biconcave disc into a spherical shape, developing a knob-like structure and finally making it lose its deformability. This results in obstruction of oxygen transport through blood circulation, i.e. capillary blockage [52].

Paper-based microfluidic platforms for its diagnosis. In a broad way, malaria is diagnosed by two methods, i.e. clinical suspicion and detection of parasites in whole blood (parasitological diagnosis) [50]. Although malaria may have varying signs and symptoms, the common clinical suspicion of malaria symptoms is a history of fever. The specificity of clinical diagnosis is very low hence it is suitable only for uncomplicated malaria. Since the epidemiology of malaria is changing continuously and it is becoming life-threatening in some cases, diagnosis of malaria with very high specificity and sensitivity is required. Parasitological diagnostic methods can fulfil the requirement of urgent and highly sensitive malaria diagnosis. RDTs and light microscopy are the most widely used methods for parasitological diagnosis. The main advantages of diagnosing malaria using light microscopy are speciation and quantification of parasites, detection of other causes of fever, measurement of the response to treatment of malaria, etc. [50]. However, light microscopy can be only used inside a laboratory and requires highly skilled people for experimentation. In many underdeveloped or undeveloped countries, most malaria patients are treated in rural areas (outside of a proper health centre) with no available resources. The use of light microscopy is not feasible in those cases. Therefore, RDTs may be the preferred choice there. The sensitivity and specificity of RDTs depend on various environmental parameters such as humidity, temperature, etc. Researchers have been continuously improving the methodologies of RDTs to eliminate such dependencies. Malaria RDTs detect specific antigens or parasite-produced proteins in infected blood. There is a significant colour change on the paper strip if there is a presence of antigens in the blood [53]. The rapid detection kits available can detect $P$. falciparum, $P$. vivax, $P$. ovale and P. malariae.

Over the past few decades, a variety of paper-based devices have been developed for the diagnosis of malaria parasites [50,51,54-62]. An automated two-dimensional paper device has been fabricated for the detection of Plasmodium falciparum histidine-rich protein 2 (PfHRP2) [54]. The paper device is made in two parts. In the first part, a nitrocellulose paper strip of 
optimized size and shape is placed, while in the second part, a CP, buffer pad and gold enhancement reagent pad are placed. A complete kit is made by folding both of these parts (Figure 9). $\mathrm{A} \mathrm{CO}_{2}$ laser beam machine is used to cut all the components into the desired shapes. An antibody to PfHRP2 is patterned at the test line and an anti-mouse antibody is printed at the control line on the paper strip. Also, a conjugated solution of a second antibody to PfHRP2 and gold nanoparticles is printed on the CP. A piezoelectric inkjet printer is used to print these patterns on paper. Gold enhancement (GE) reagent is used for amplification of the signal. The limit of detection of the device for detection of $P$. falciparum is $2.9 \pm 1.2 \mathrm{ng} \mathrm{mL}^{-1}$.

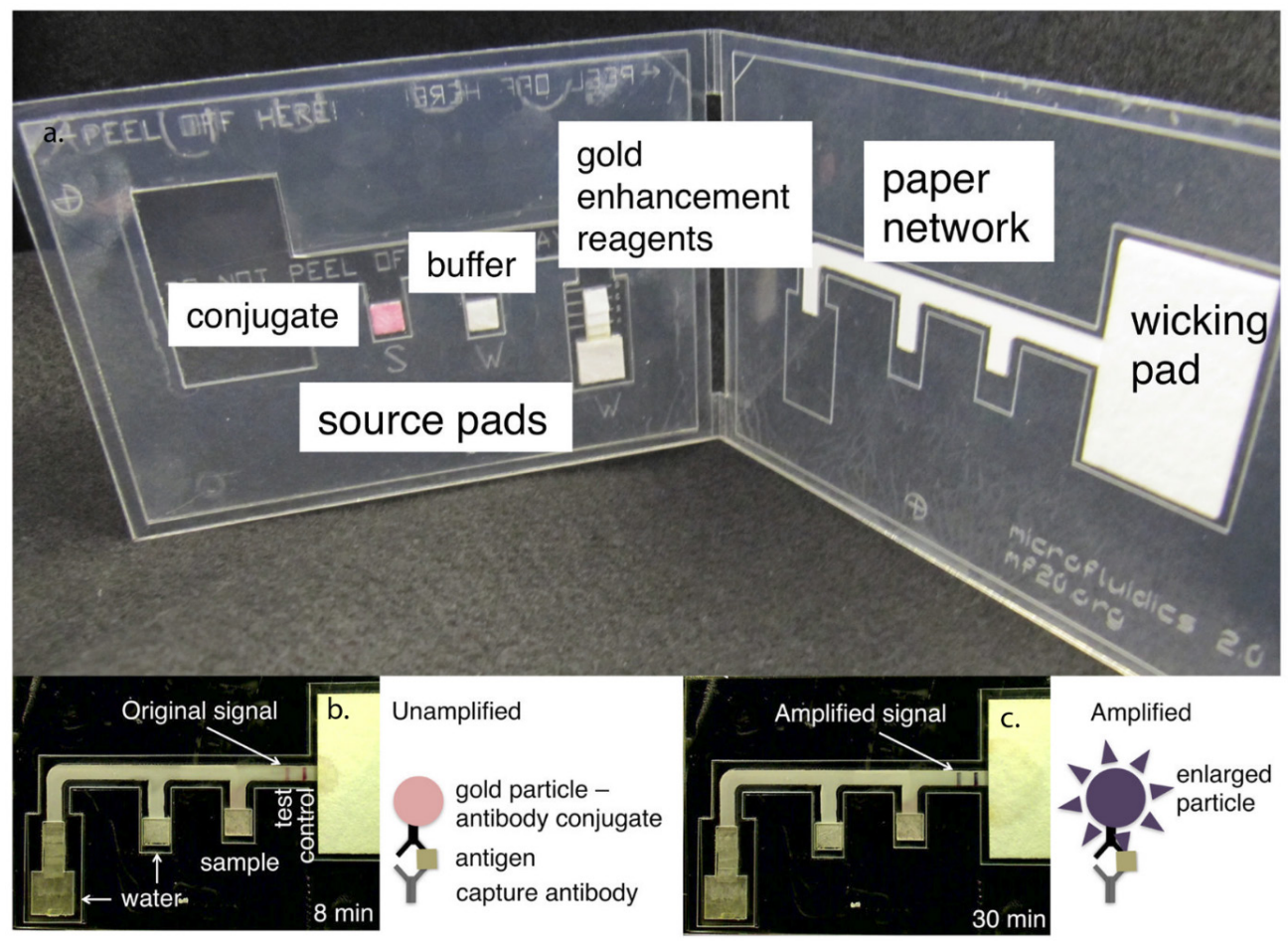

Figure 9. Two-dimensional paper network for detection of P.f., (a) two-dimensional lateral flow strip, (b) Original pink color signal at test line if GE reagent is not used, (c) dark purple signal at test line when GE reagent is used. Reprinted with permission from E. Fu et al. [54], Two-dimensional paper network format that enables simple multistep assays for use in low-resource settings in the context of malaria antigen detection, Anal. Chem. 84 (2012) 4574-4579. ๑2016 American Chemical Society.

In order to reduce the limit of detection, a hybrid paper-based diagnostic device has been fabricated for the detection of a malaria biomarker, Plasmodium lactate dehydrogenase (pLDH) [59]. pLDH is a glycolytic enzyme which forms in the asexual stages of malaria parasites. The device is comprised of two systems, i.e. a lateral flow immunoassay (LFA) and a micellar aqueous 
two-phase system (ATPS). The device is capable of simultaneous concentration and detection of pLDH in both phosphate-buffered saline (PBS) and fetal bovine serum (FBS). The same protocol is used for the detection of pLDH in both systems with the exception of using a different volumetric ratio of chemicals in the preparation of ATPS. A known amount of ATPS and antipLDH-gold nanoparticles are mixed in a test tube and then a known concentration of pLDH is added to it. This solution is incubated for $5 \mathrm{~min}$ for phase separation as well as capturing of pLDH by gold nanoparticles. The LFA strip is then dipped vertically into the sample solution. The solution starts flowing through the paper strip by capillary action and reaches the test line where the colour band is formed. A schematic diagram of the paper-based detection device is shown in Figure 10.

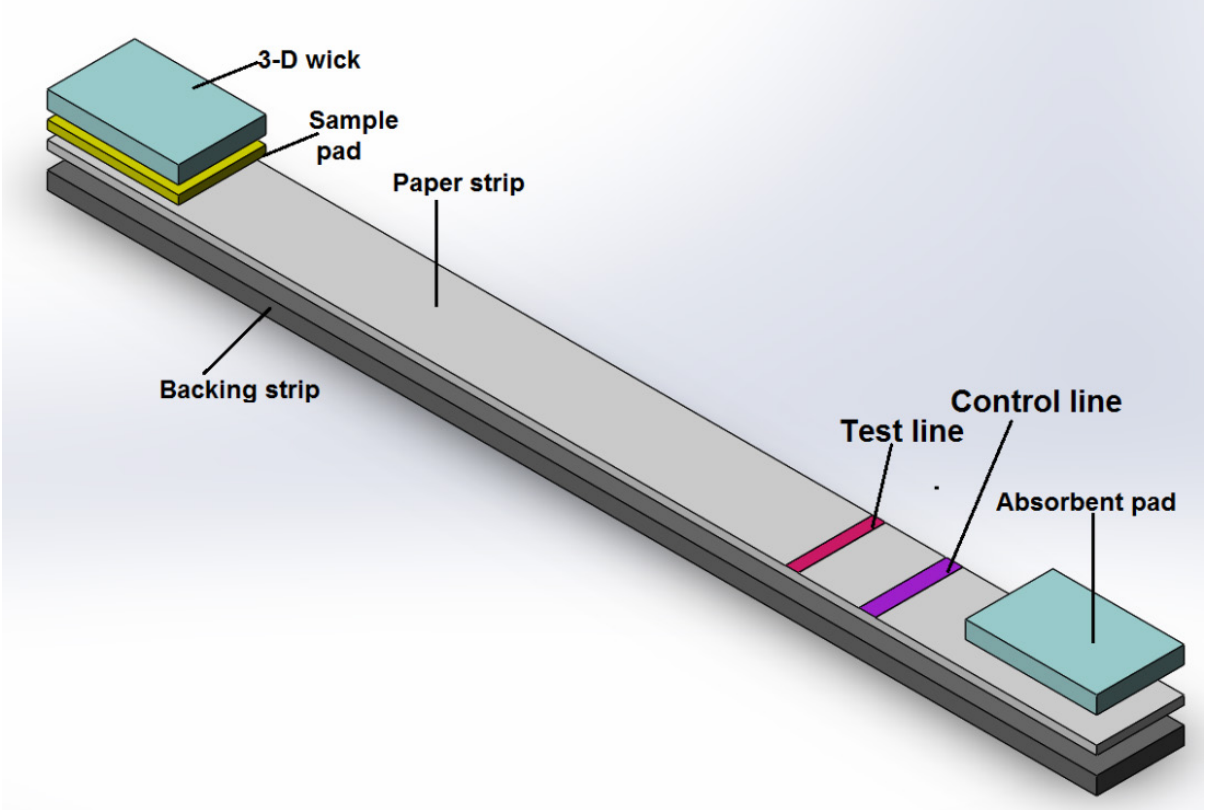

Figure 10. Schematic of a single-step paper-based detection device for malaria pLDH detection

Deraney et al. [63] reported a patterned paper immunoassay for detection of two malaria antigens, malaria HRP2 and malaria pLDH. They developed a three-dimensional paper-based microfluidic device (Figure 11) capable of detecting both malaria antigens with high sensitivity which can also distinguish between the two malaria species. 


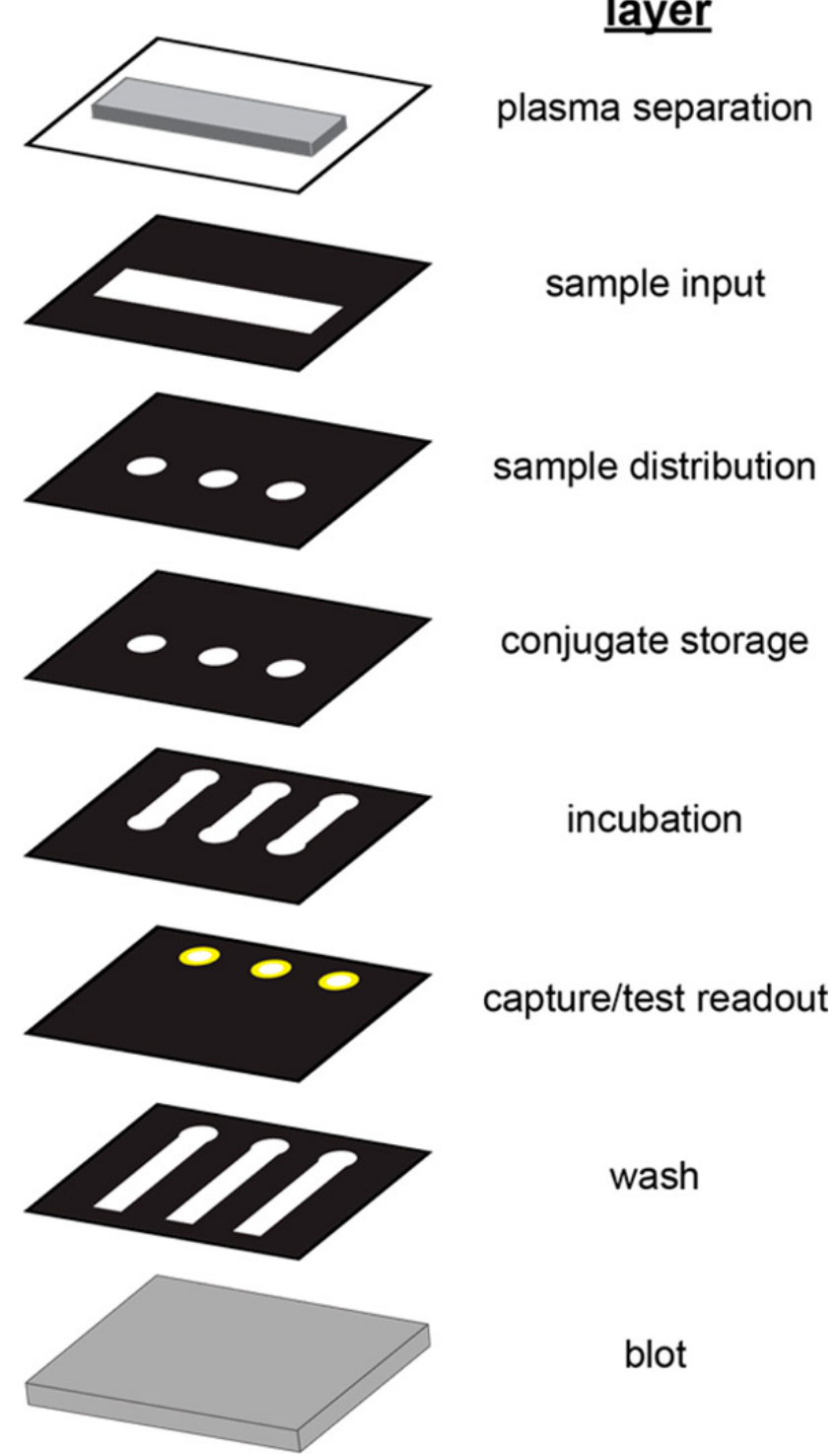

Figure 11. Schematic of a three-dimensional paper-based device used for detection of malaria antigens. Reprinted with permission from Deraney et al. ${ }^{\circ} 2016$ American Chemical Society.

For the purpose of amplification and detection of malaria, a self-sealing paper and plastic system has been fabricated [64]. The detection of Plasmodium is based on nucleic acid tests. The primary function of the developed paper and plastic device is to amplify the DNA sequence using recombinase polymerase amplification (RPA), dilute the resultant amplified product repeatedly and detect Plasmodium DNA using a paper-based assay. In order to carry out these 
functions, a sequence of specific reagent-loaded paper pads is used (Figure 12). The amplification function is carried out on an RPA pad (made of Whatman filter paper grade 1), the dilution process is carried out in four steps using four different pads (made of blotter paper) and the detection of Plasmodium DNA is carried out on a lateral flow strip. The developed device is user-friendly and the total time taken for detection of malaria virus is reported to be $1 \mathrm{~h}$.

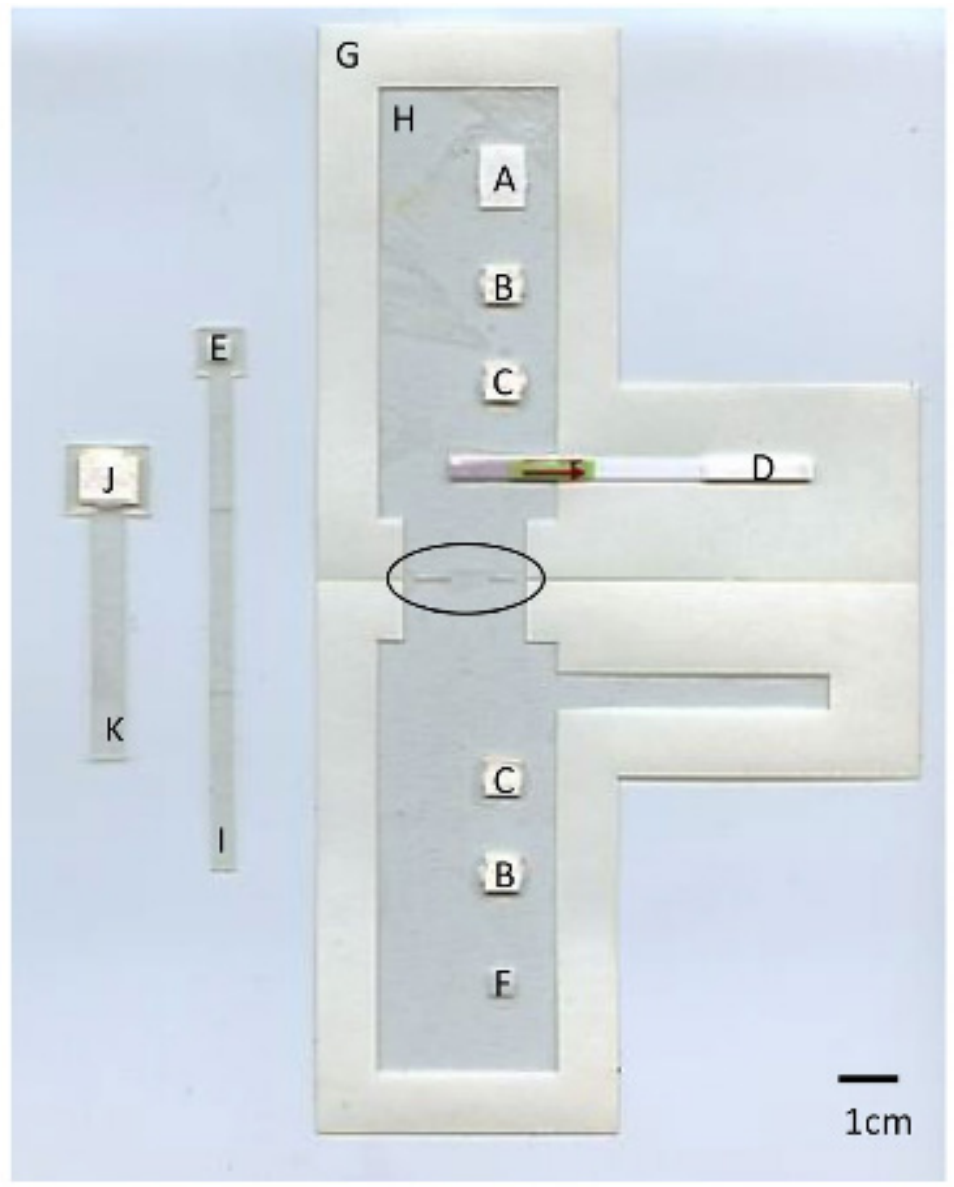

Figure 12. Schematic of a paper and plastic amplification and detection device. Reprinted with permission from Cordray et al. [64], ${ }^{\odot 2015}$ Cordray and RichardsKortum.

Although paper-based immunoassays are widely used for colorimetric diagnostic tests for malaria, the sometimes unreliable appearance of colour on the strip makes it very difficult to interpret the result. To overcome this problem, Lathwal and Sikes [65] developed an advanced colorimetric method to detect the malaria biomarker PfHRP2. The colorimetric signal of the fabricated paper-based device is amplified by a photoinitiated polymerization 
reaction. The images of the amplified results are captured with a cellphone for RGB colour analysis and calculation of the limit of detection.

Inexpensive personal paper-based diagnostic devices can aid in providing early warnings for massive outbreaks of malaria. They can be used as initial screening tools to give a rapid readout within $10 \mathrm{~min}$ or less. Some of the inadequacies such as low sensitivity and specificity, dependence on environmental factors, etc. still need to be addressed in order to enable these devices to be used on a mass scale.

\subsubsection{HIV infection}

HIV infection is a viral disease caused by the human immunodeficiency virus (HIV). An HIV infection may further lead to the development of acquired immunodeficiency syndrome (AIDS). HIV begets a progressive deterioration of the immune system by predominantly affecting the CD4 cells (T cells), allowing infections and cancers to thrive within the body. It can spread via bodily fluids like semen, vaginal fluid, pre-seminal fluid, breast milk or blood. Transmission occurs through sexual intercourse or by sharing needles or syringes [66]. The source of this virus is a breed of chimpanzee found in Central Africa [67]. The infection has three stages: acute HIV infection (display of flu-like symptoms), clinical latency (HIV stays dormant producing very low levels) and AIDS (severely damaged immune system, with a life expectancy of 3 years). No effective cure exists for HIV infection to date, but with technological and medical advancements the virus can be suppressed for long periods of time.

Causative agent. HIV belongs to the genus Lentivirus from the Retroviridae family of viruses. It gets transmitted as a single-stranded enveloped RNA virus, but converts into double-stranded DNA utilizing a reverse transcriptase upon entry into the cell. HIV-1 and HIV-2 are the two types of HIV that have been characterized. HIV-1 is the chief vector of the majority of infections, whereas HIV-2, owing to its poor transmission capability, doesn't cause many infections [68]. The virus incubates for $2-4$ weeks, after which symptoms like body rash, fever, sore throat and severe headaches start to surface. The number of CD4 cells drops drastically in the third stage (AIDS), accompanied by chills, fever, swollen lymph glands, weakness and weight loss.

Paper-based microfluidic platforms for its diagnosis. An integrated paperbased molecular diagnostic device for sensitive nucleic acid (NA) detection of HIV has been developed by Liu [69]. The device uses whole blood as the sample and performs an on-chip plasma separation within 5 min using a highly efficient plasma generation membrane. Subsequently, using a lyophilization process, the chemical components for NA amplification are embedded onto a nitrocellulose (NC) membrane. As a result, florescence intensity readout of the NC membrane is taken after 20 min of NA amplification. A limit of detection of 10 copies has been reported for this device. Zhao and Liu have developed a portable paper-based microfluidic platform for the detection of HIV in human 
serum [70]. The platform has the capability to perform indirect ELISA simultaneously on eight samples within $20 \mathrm{~min}$. An electrochemical immunoassay array and handheld potentiostat based on a microcontroller unit are used for taking readings. The platform also integrates a wireless communication module in its design for easy transfer of results to aid patients in remote areas. The sensitivity of the device using mouse serum samples is reported to be $300 \mathrm{pg} \mathrm{mL}^{-1}$.

Electrical sensing using printed graphene-modified electrodes on a cellulose paper has been done by Safavieh etal. for the detection of HIV-1 [71]. A schematic of the microchip illustrating the virus capture process and its lysis is given in Figure 13. The effect of surface chemistry is investigated by immobilizing anti-gp120 antibodies on the cellulose pads, with and without streptavidin. The microchip uses an HIV-spiked sample and after $30 \mathrm{~min}$ impedance/capacitance spectroscopy is carried out using an LCR meter for frequencies in the range of 1-10 kHz. Streptavidin-coated chips show better sensitivity by demonstrating a five times increase in the capacitance value.

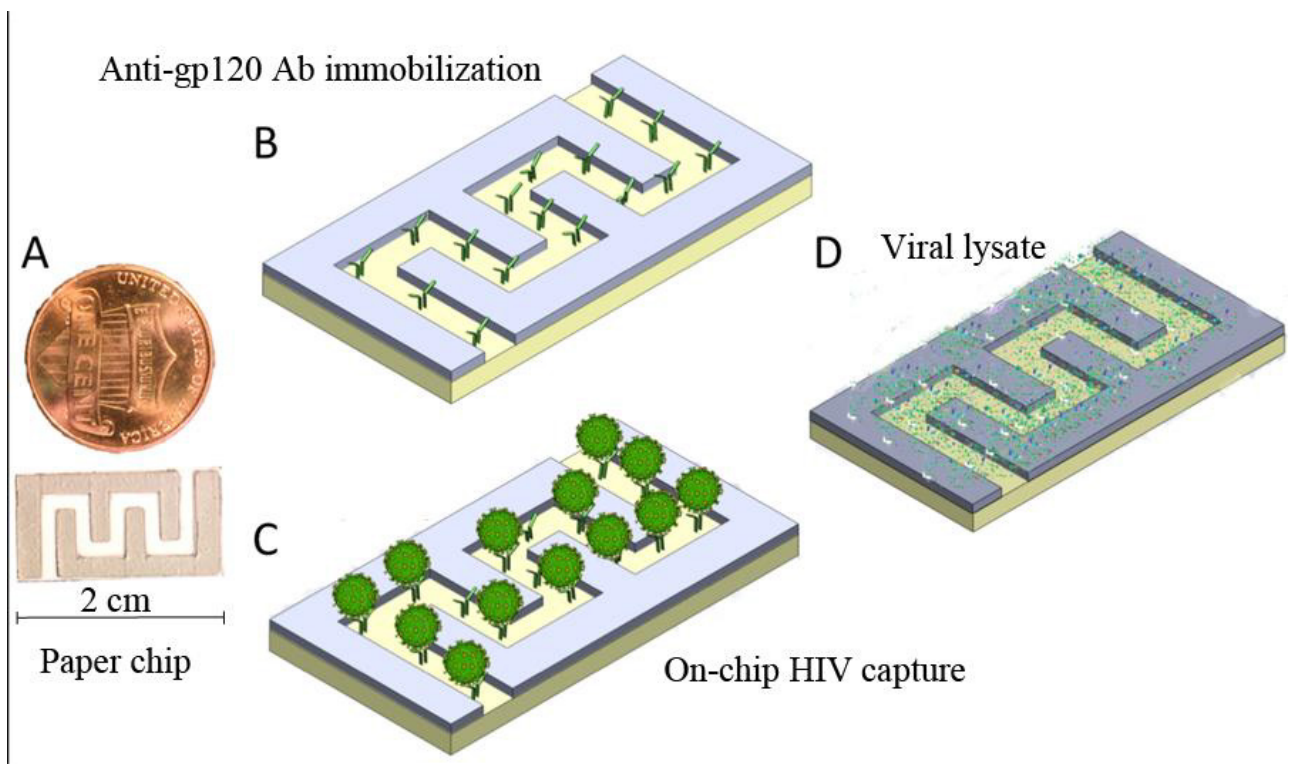

Figure 13. Schematic of virus capture and on-chip detection. (A) Fabricated paper microchip, (B) paper substrate immobilized with anti-gp120 Ab, (C) virus captured on the paper chip, (D) lysis of captured viruses. Reprinted with permission from Safavieh et al. [71]. ${ }^{\circledR 2016}$ SPIE.

Rohrman and Richards-Kortum have fabricated a paper and plastic device for carrying out isothermal, enzymatic amplification of HIV DNA [72]. The device exploits RPA for NA amplification of HIV (Figure 14). Different materials such as cellulose, glass fibre, Fusion 5 , etc. were experimentally tested to select the optimal substrate for RPA. Glass fibre exhibited the least noise and maximum product and was hence chosen for developing the device. 


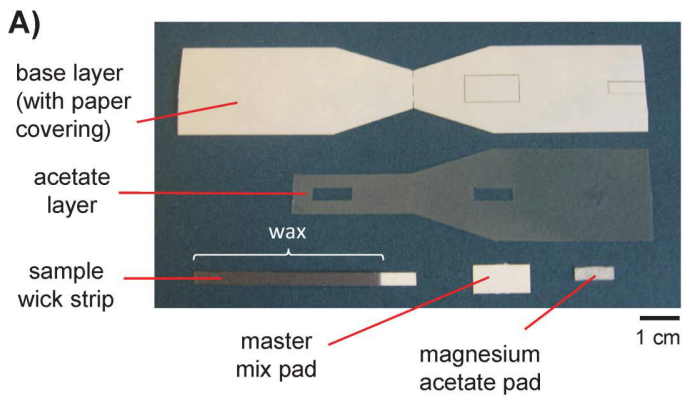

B)

1. place enzyme pellet on exposed adhesive

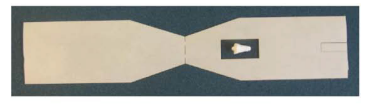

2. place master mix pad and sample wick strip

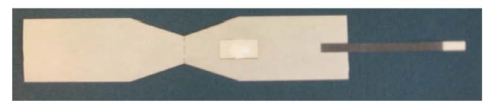

3. remove remaining paper to expose adhesive

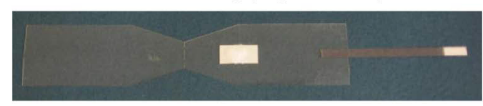

4. place acetate layer over exposed adhesive

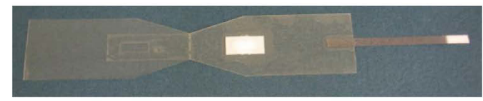

5. place magnesium acetate pad in window

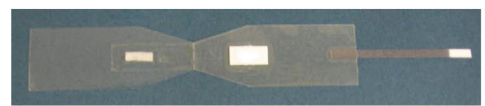

C) 1. add master mix

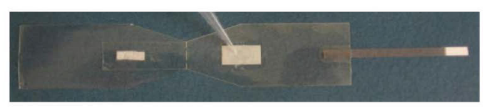

2. add magnesium acetate

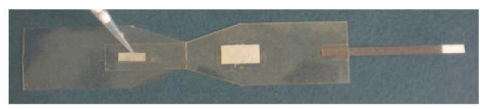

3. dip wick in sample

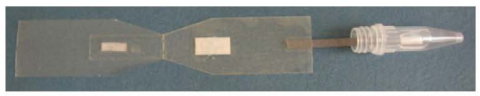

4. fold wick

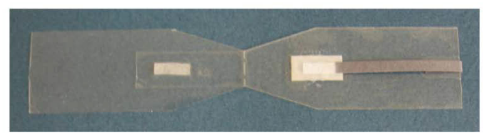

5. fold device in half

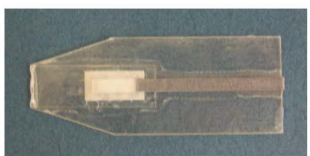

Figure 14. Schematic of paper and plastic device for RPA of HIV DNA. (A) Components of the device, (B) assembly of the device, (C) device operation. Reprinted with permission from Rohrman et al. [72]. ${ }^{\circ}$ Royal Society of Chemistry.

Next, commercially available lateral flow assays were used to detect the products of RPA. Gold nanoparticles were conjugated to anti-FAM antibodies, immobilized on a CP and anti-biotin antibodies on the test line. The device can amplify 10 copies of HIV with a detection time of $15 \mathrm{~min}$.

Lateral flow assays have dominated rapid diagnosis of HIV infection. Their compactness and portability makes them accessible to patients in remote areas with few resources, but they are still in their early stages and challenges like scaling up, commercialization, etc. have yet to be addressed.

\subsubsection{Zika}

Zika is a viral disease first identified in Uganda in 1947 in rhesus monkeys and in 1952 in humans. It spread as an epidemic in early 2015, leading the WHO to 
declare it a public health emergency. Its potential to cause fetal neurodevelopmental issues [73,74] and Guillain-Barré syndrome [75] has made it indispensable to develop effective, rapid and low-cost diagnostic platforms. Such field-based diagnostic platforms can help mitigate the need for laboratory testing.

Causative agent. Zika is spread by the bite of infected A. aegypti and $A$. albopictus mosquitoes. The virus belongs to the Flaviviridae family, the same as dengue, yellow fever, chikungunya, etc. The symptoms of the virus are typically visible within 2-7 days. The symptoms include fever, muscle and joint pain, headache, conjunctivitis, etc.

Paper-based microfluidic platforms for its diagnosis. Recently, a novel portable, low-cost paper-based diagnostic platform for Zika virus has been developed by researchers [76]. The technique utilizes viremic plasma for detection and is capable of providing results within $3 \mathrm{~h}$ with sensitivity in the femtomolar (fM) range. The device follows a workflow involving sensor development, Zika virus detection and strain identification (Figure 15).

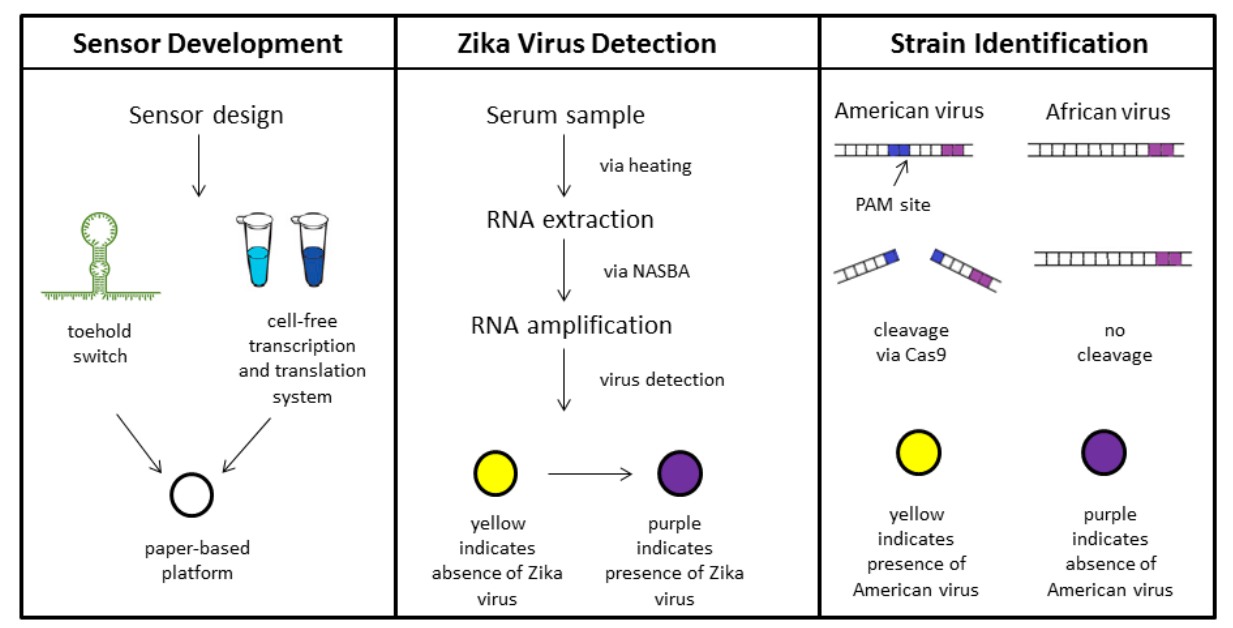

Figure 15. Schematic representation of the device workflow

The sensor development stage involves assembly of the sequence-specific toehold switch-based sensors followed by their embedding onto paper. The amplification and sensor design process has been automated using in silico algorithms enabling the system to test eight Zika sensors in $7 \mathrm{~h}$. These paperbased platforms are then freeze-dried along with a cell-free transcription and translation system. The assays are then deployed in the field for detection purposes. Zika virus detection is performed by rehydrating the paper platform with a serum sample, and monitoring a colour change from yellow to purple. The fabricated sensor was found to be specific for Zika virus by testing it 
against substantially similar dengue genome sequences. The sensor accommodates isothermal nucleic acid sequence-based amplification (NASBA) for sensitivity improvement. The last stage of the sensor focuses on discrimination of Zika strains against other identical genomes using a NASBA-CRISPR (clustered regularly interspaced short palindromic repeats) cleavage assay. The assay has been validated to distinguish between dengue and Zika sequences and American and African Zika strains. The sensor validation was done in steps by utilizing live Zika virus, i.e. Uganda strain MR 766. Subsequently, active Zika virus was spiked at a concentration of $10 \mathrm{fM}$ into a human blood serum sample and successfully tested using the sensor. The final validation of the sensor was done by using viremic macaque plasma infected with Zika virus. The plasma was diluted, heated and then amplified via NASBA. Strong activation of the sensor was observed.

Paper-based detection platforms show promise for vector control of Zika virus. However, extensive research is required to assess the validity of such developed techniques on affected human samples, to further improve the sensitivity and achieve much faster detection rates.

\subsection{SUMMARY}

Over the last decade, the domain of paper-based diagnostics has expanded immensely. Their low cost, portability, ease of use and flexibility make them promising for the development of rapid diagnostic assays. In view of the technological advancements made in this field, it is possible to envision the utility of paper-based POC diagnostic devices for rapid and accurate diagnosis of several diseases on a mass scale. However, many important issues need to be considered to realize the commercialization of these devices such as their stability, accurate quantification, specificity and selectivity. Given the progress made, it can be established that the future of disease diagnostics using paperbased platforms presents a lot of potential. 


\section{REFERENCES}

1. J. Chin \& A.P.H. Association, Control of communicable diseases manual, American Public Health Association Washington, DC, USA, 2000.

2. N. Mohita. 9 Classifications of Communicable Diseases according to Nature of the Pathogen, <http://www.yourarticlelibrary.com/disease/9-classifications-ofcommunicable-diseases-according-to-nature-of-the-pathogen/14185/> (2015).

3. M.P. Taylor, O. Kobiler, L.W. Enquist. Proc. Natl. Acad. Sci. USA 109 (2012) 17046-17051.

4. Viral Diseases, <https://www.healthgrades.com/conditions/viral-diseases $>$ (2013).

5. K.T. Andrews, G. Fisher, T.S. Skinner-Adams. Int. J. Parasitol. Drugs Drug Resist. 4 (2014) 95-111.

6. W.G. Lee, Y.-G. Kim, B.G. Chung, U. Demirci, A. Khademhosseini. Adv. Drug Deliv. Rev. 62 (2010) 449-457.

7. A. Hamraoui, T. Nylander., J. Colloid Interface Sci. 250 (2002) 415-421.

8. H. Hwang, S.-H. Kim, T.-H. Kim, J.-K. Park, Y.-K. Cho. Lab Chip 11 (2011) 3404-3406.

9. F.A. Dullien, Porous media: fluid transport and pore structure, Academic press, 2012.

10. S. Mendez, E.M. Fenton, G.R. Gallegos, D.N. Petsev, S.S. Sibbett, H. A. Stone, Y. Zhang, G.P. López. Langmuir 26 (2009) 1380-1385.

11. M. Santhiago, E.W. Nery, G.P. Santos, L.T. Kubota. Bioanalysis 6 (2014) 89-106.

12. $\quad$ E. Elizalde, R. Urteaga, C.L. Berli. Lab Chip 15 (2015) 2173-2180.

13. X. Wang, J.A. Hagen, I. Papautsky. Biomicrofluidics 7 (2013) 014107.

14. S. Kumar, P. Bhushan, S. Bhattacharya. Anal. Methods (2016) doi:10.1039/C6AY01926A.

15. R. Jabara, N. Chronos, D. Conway, W. Molema, K. Robinson. JACC Cardiovasc. Interv. 1 (2008) 81-87.

16. J. Nie, Y. Liang, Y. Zhang, S. Le, D. Li, S. Zhang. Analyst 138 (2013) 671-676.

17. J.L. Delaney, C.F. Hogan, J. Tian, W. Shen. Anal. Chem. 83 (2011) 1300-1306.

18. X. Li, J. Tian, G. Garnier, W. Shen. Colloids Surf. B: Biointerfaces 76 (2010) 564-570.

19. X. Li, J. Tian, W. Shen. Cellulose 17 (2010) 649-659.

20. Y. Lu, W. Shi, L. Jiang, J. Qin, B. Lin. Electrophoresis 30 (2009) 1497-1500.

21. E. Carrilho, A.W. Martinez, G.M. Whitesides. Anal. Chem. 81 (2009) 7091-7095.

22. J. Olkkonen, K. Lehtinen, T. Erho. Anal. Chem. 82 (2010) 10246-10250.

23. S.A. Klasner, A.K. Price, K.W. Hoeman, R.S. Wilson, K.J. Bell, C.T. Culbertson. Anal. Bioanal. Chem. 397 (2010), 1821-1829.

24. T. Songjaroen, W. Dungchai, O. Chailapakul, W. Laiwattanapaisal. Talanta 85 (2011) 2587-2593.

25. X. Li, J. Tian, T. Nguyen, W. Shen. Anal. Chem. 80 (2008) 9131-9134.

26. T. Nurak, N. Praphairaksit, O. Chailapakul. Talanta 114 (2013) 291-296.

27. J. Nie, Y. Zhang, L. Lin, C. Zhou, S. Li, L. Zhang, J. Li. Anal. Chem. 84 (2012) 6331-6335.

28. D.M. Cate, J.A. Adkins, J. Mettakoonpitak, C.S. Henry. Anal. Chem. 87 (2014) 19-41. 
29. K.F. Lei, Chapter 1 :Materials and fabrication techniques for nano-and microfluidic devices, 2014, 1-28.

30. R.L.F.T. Strips, Bedford, MA: Millipore Corporation, (2001).

31. C.-W. Yen, H. de Puig, J.O. Tam, J. Gómez-Márquez, I. Bosch, K. Hamad-Schifferli, L. Gehrke. Lab Chip 15 (2015) 1638-1641.

32. M.G. Guzman, S.B. Halstead, H. Artsob, P. Buchy, J. Farrar, D.J. Gubler, E. Hunsperger, A. Kroeger, H.S. Margolis, E. Martínez. Nature Rev. Microbiol. 8 (2010) S7-S16.

33. G.P. Ashutosh Biswas, V. Devgan, P. Singla, P. Murthy, A.C. Dhariwal, P.K. Sen, K. Baruah. J. Indian Med. Assoc. 113 (2015) 196-206.

34. W.H. Organization, S.P.f. Research, T.i.T. Diseases, W.H.O.D.o.C.o.N.T. Diseases, W.H.O. Epidemic and P. Alert, Dengue: guidelines for diagnosis, treatment, prevention and control, World Health Organization, 2009.

35. S.-J. Lo, S.-C. Yang, D.-J. Yao, J.-H. Chen, W.-C. Tu, C.-M. Cheng, Lab Chip 13 (2013) 2686-2692.

36. B. Zhang, G.B. Salieb-Beugelaar, M.M. Nigo, M. Weidmann, P. Hunziker. Nanomedicine 11 (2015) 1745-1761.

37. R.W. Peeling, H. Artsob, J.L. Pelegrino, P. Buchy, M.J. Cardosa, S. Devi, D.A. Enria, J. Farrar, D.J. Gubler, M.G. Guzman. Nature Rev. Microbiol. 8 (2010) S30-S37.

38. K.H. Sellahewa. ISRN Infectious Diseases 2013 (2012).

39. S.M. Wang, D. Sekaran. Am. J. Trop. Med. Hyg. 83 (2010) 690-695.

40. Y. Zhang, J. Bai, J.Y. Ying. Lab Chip 15 (2015) 1465-1471.

41. P. Yager, E. Fu, T. Liang, B. Lutz, J.L. Osborn. 15th International Conference on Miniaturized Systems for Chemistry and Life Sciencesm, October 2-6, 2011, Seattle, Washington, USA.,

42. J. Matthews, R. Kulkarni, M. Gerla, T. Massey. Mobile Netw. Appl. 17 (2012) 178-191.

43. E.M. Leroy, B. Kumulungui, X. Pourrut, P. Rouquet, A. Hassanin, P. Yaba, A. Délicat, J.T. Paweska, J.-P. Gonzalez, R. Swanepoel. Nature 438 (2005) 575-576.

44. S.F. Dowell, R. Mukunu, T.G. Ksiazek, A.S. Khan, P.E. Rollin, C. Peters. J. Infect. Dis. 179 (1999) S87-S91.

45. H. Feldmann, T.W. Geisbert. Lancet 377 (2011) 849-862.

46. J.S. Towner, P.E. Rollin, D.G. Bausch, A. Sanchez, S.M. Crary, M. Vincent, W.F. Lee, C.F. Spiropoulou, T.G. Ksiazek, M. Lukwiya. J. Virol. 78 (2004) 4330-4341.

47. T.G. Ksiazek, C.P. West, P.E. Rollin, P.B. Jahrling, C. Peters. J. Infect. Dis. 179 (1999) S192-S198.

48. K. Pardee, A.A. Green, T. Ferrante, D.E. Cameron, A. Daley Keyser, P. Yin, J.J. Collins. Cell 159 (2014) 940-954.

49. ReEBOV Antigen Rapid Test <ftp://ftp.corgenix.com/Intra/ReEBOV/14005.pdf> (2016).

50. H. Reyburn. New WHO guidelines for the treatment of malaria. Report No. 0959-8138, c2637 (2010).

51. M.P. Horning, C.B. Delahunt, S.R. Singh, S.H. Garing, K.P. Nichols. Lab Chip 14 (2014) 2040-2046.

52. J.P. Shelby, J. White, K. Ganesan, P.K. Rathod, D.T. Chiu. Proc. Natl. Acad. Sci. USA 25 (2003) 14618-14622. 
53. D. Bell, R.W. Peeling. Nature Rev. Microbiol. 4 (2006) S34-S38.

54. E. Fu, T. Liang, P. Spicar-Mihalic, J. Houghtaling, S. Ramachandran, P. Yager. Anal. Chem. 84 (2012) 4574-4579.

55. W. Jeon, S. Lee, D. Manjunatha, C. Ban. Anal. Biochem. 439 (2013) 11-16.

56. S. Jones, C.J. Sutherland, C. Hermsen, T. Arens, K. Teelen, R. Hallett, P. Corran, M. van der Vegte-Bolmer, R. Sauerwein, C.J. Drakeley. Malar. J. 11 (2012) 1.

57. A. Moody. Clin. Microbiol. Rev. 15 (2002) 66-78.

58. W.H. Organization. Malaria rapid diagnostic test performance: results of WHO product testing of malaria RDTs: round 6 (2014-2015). Report No. 924151003X, (2015).

59. D.Y. Pereira, R.Y. Chiu, S.C. Zhang, B.M. Wu, D.T. Kamei. Anal. Chim. Acta 882 (2015) 83-89.

60. J.P. Shelby, J. White, K. Ganesan, P.K. Rathod, D.T. Chiu. Proc. Natl. Acad. Sci. USA 100 (2003) 14618-14622.

61. A.A. Weaver, M. Lieberman. Am. J. Trop. Med. Hyg. 92 (2015) 17-23.

62. J. Wu, Y. Peng, X. Liu, W. Li, S. Tang. Acta Parasitologica 59 (2014) 267-271.

63. R.N. Deraney, C.R. Mace, J.P. Rolland, J.E. Schonhorn. Anal. Chem. (2016).

64. M.S. Cordray, R.R. Richards-Kortum. Malar. J. 14 (2015) 1.

65. S. Lathwal, H.D. Sikes. Lab Chip 16 (2016) 1374-1382.

66. R. Health, A. Services, C.f.D. Control, Prevention and H.M.A.o.t.I.D.S.o. America, MMWR. Recommendations and reports: Morbidity and mortality weekly report. Recommendations and reports/Centers for Disease Control 52 (2003) 1.

67. E. Hooper, The river: a journey to the source of HIV and AIDS, MA: Allen LanePenguin Press/Little, Brown \& Co., 2000.

68. A. Rambaut, D. Posada, K.A. Crandall, E.C. Holmes. Nat. Rev. Genet. 5 (2004) 52-61.

69. F. Liu. Biophys. J. 106 (2014) 417a.

70. C. Zhao, X. Liu. Biomicrofluidics 10 (2016) 024119.

71. M. Safavieh, S. Khetani, V. Kaul, D.R. Kuritzkes, H. Shafiee, in SPIE Sensing Technology+ Applications, A graphene-modified cellulose paper microchip for HIV detection, International Society for Optics and Photonics, 94900G-94900G94907.

72. B.A. Rohrman, R.R. Richards-Kortum. Lab Chip 12 (2012) 3082-3088.

73. G. Calvet, R.S. Aguiar, A.S. Melo, S.A. Sampaio, I. de Filippis, A. Fabri, E.S. Araujo, P.C. de Sequeira, M.C. de Mendonça, L. de Oliveira. Lancet Infect. Dis. (2016).

74. J. Mlakar, M. Korva, N. Tul, M. Popović, M. Poljšak-Prijatelj, J. Mraz, M. Kolenc, K. Resman Rus, T. Vesnaver Vipotnik, V. Fabjan Vodušek. N. Engl. J. Med. 374 (2016) 951-958.

75. E. Oehler, L. Watrin, P. Larre, I. Leparc-Goffart, S. Lastere, F. Valour, L. Baudouin, H. Mallet, D. Musso, F. Ghawche. Euro Surveill. 19 (2014) 20720.

76. K. Pardee, A.A. Green, M.K. Takahashi, D. Braff, G. Lambert, J.W. Lee, T. Ferrante, D. Ma, N. Donghia, M. Fan. Cell 165 (2016) 1255-1266. 
Chapter 2 